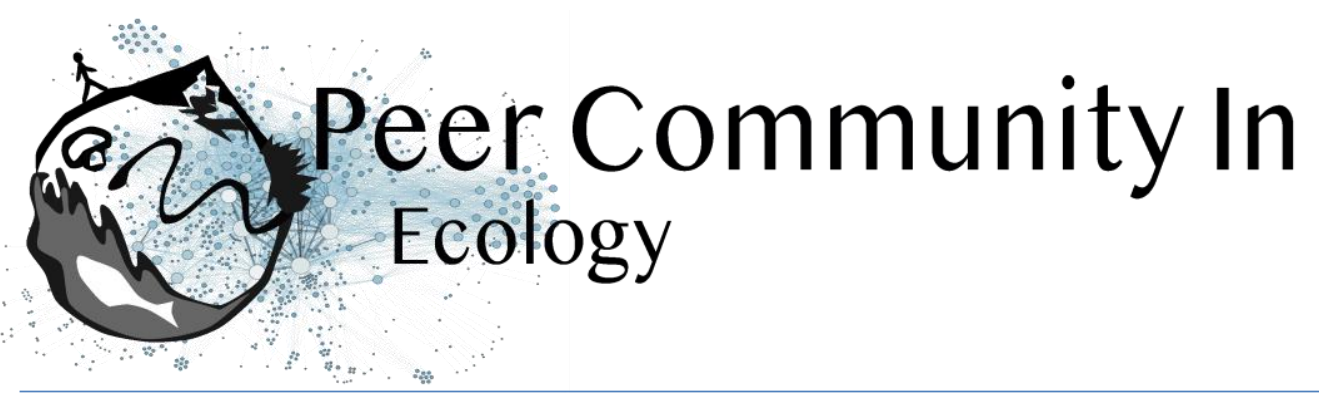

\title{
Blue tits surviving in an ever-changing world
}

Dieter Lukas based on reviews by Vicente García-Navas and Ana Sanz-Aguilar

\section{A recommendation of:}

\author{
Identifying drivers of spatio-temporal variation in survival in four blue tit \\ populations \\ Olivier Bastianelli, Alexandre Robert, Claire Doutrelant, Christophe de Franceschi, Pablo \\ Giovannini, Anne Charmantier (2021), bioRxiv, 2021.01.28.428563, ver. 4 peer-reviewed \\ and recommended by Peer community in Ecology 10.1101/2021.01.28.428563
}

Submitted: 29 January 2021, Recommended: 28 May 2021

\section{Open Access}

Published: 02 June 2021

Copyright: This work is licensed under the Creative Commons Attribution-NoDerivatives 4.0 International License. To view a copy of this license, visit http:// creativecommons.org/licen ses/by-nd/4.0/
Cite this recommendation as:

Dieter Lukas (2021) Blue tits surviving in an ever-changing world. Peer Community in Ecology, 100085. 10.24072/pci.ecology.100085

\section{Recommendation}

How long individuals live has a large influence on a number of biological processes, both for the individuals themselves as well as for the populations they live in. For a given species, survival is often summarized in curves showing the probability to survive from one age to the next. However, these curves often hide a large amount of variation in survival. Variation can occur from chance, or if individuals have different genotypes or phenotypes that can influence how long they might live, or if environmental conditions are not the same across time or space. Such spatiotemporal variations in the conditions that individuals experience can lead to complex patterns of evolution (Kokko et al. 2017) but because of the difficulties to obtain the relevant data they have not been studied much in natural populations.

In this manuscript, Bastianelli and colleagues (2021) identify which environmental and population conditions are associated with variation in annual survival of blue tits. The analyses are based on an impressive dataset, tracking a total of almost 5500 adults in four populations studied for at least 19 years. The authors describe two core results. First, average annual survival is lower in deciduous forests compared to evergreen forests. The differences in average annual survival between the forest types link with previously described differences, with individuals having larger clutches (Charmantier et al. 2016) and higher aggression (Dubuc-Messier et al. 2017) in the populations where adult survival is lower. Second, there are huge fluctuations from one year to the next in the percentage of individuals surviving which occur similarly in all populations. Even though survival covaried across the four populations, this variation was not associated with any of the local or global climate indices the authors investigated.

Studies like these are fundamental to our understanding of population change. They are important from an applied side as they can reveal the sustainability of populations and inform potential management options. On a basic research side, they reveal how evolution operates in populations. Theoretical studies predict that individuals are often 


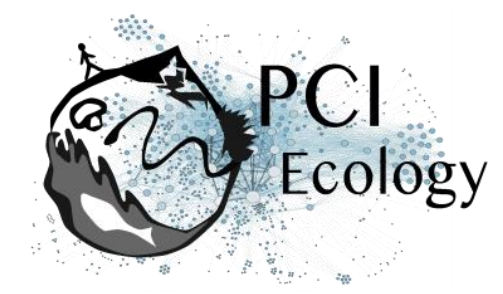

not adapted to average conditions they experience, but either selected to balance the extremes they encounter or to make the best during harsh conditions when it really matters (Lewontin \& Cohen 1969).

This study also opens the door to new research, highlighting that demographic studies should pay attention to variation in survival and other life history traits. For blue tits specifically, the study shows that in order to understand the demography of populations we need a better mechanistic understanding of the environmental and physiological pressures influencing whether individuals die or not to make predictions whether and how climate or other ecological effects shape variation in survival.

\title{
References
}

Bastianelli O, Robert A, Doutrelant C, Franceschi C de, Giovannini P, Charmantier A (2021) Identifying drivers of spatio-temporal variation in survival in four blue tit populations. bioRxiv, 2021.01.28.428563, ver. 4 peerreviewed and recommended by Peer community in Ecology. https://doi.org/10.1101/2021.01.28.428563

Charmantier A, Doutrelant C, Dubuc-Messier G, Fargevieille A, Szulkin M (2016) Mediterranean blue tits as a case study of local adaptation. Evolutionary Applications, 9, 135-152. https://doi.org/10.1111/eva.12282

Dubuc-Messier G, Réale D, Perret P, Charmantier A (2017) Environmental heterogeneity and population differences in blue tits personality traits. Behavioral Ecology, 28, 448459. https://doi.org/10.1093/beheco/arw148

Kokko H, Chaturvedi A, Croll D, Fischer MC, Guillaume F, Karrenberg S, Kerr B, Rolshausen G, Stapley J (2017) Can Evolution Supply What Ecology Demands? Trends in Ecology \& Evolution, 32, 187197. https://doi.org/10.1016/j.tree.2016.12.005

Lewontin RC, Cohen D (1969) On Population Growth in a Randomly Varying Environment. Proceedings of the National Academy of Sciences, 62, 1056-1060. https://doi.org/10.1073/pnas.62.4.1056

\section{Reviews}

Toggle reviews

\section{Revision round \#2}

\author{
2021-05-18
}

\section{Author's Reply}

Download author's reply (PDF file)Download tracked changes file You will attached our response letter, as well as the tracked changed document. All other documents (data and main revised manuscript file) have been submitted as revisions in BioRxiv.

Best regards,

Ann Charmantier

\section{Decision round \#2}

Dear authors, 


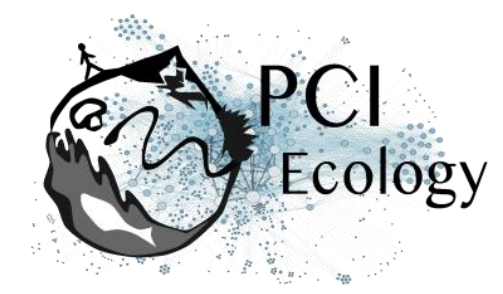

Thank you for addressing all the comments of the reviewers and me in such detail. The manuscript now contains additional details helpful to fully appreciate the inferences your study provides. I only have a few, very minor suggestions for changes that should be easy to address so I can recommend this study.

Comparison with previous study by Grosbois and colleagues:

In the introduction, there is a bit of a jump from the pace-of-life arguments (paragraph 105-132) to the study by Grosbois (lines 133-143) back to your framing around the pace-of-life (paragraph 144-150). I think it would help to see more clearly how the study by Grosbois et al fits within the frame of your study. Did Grosbois and colleagues also look at factors related to the pace-of-life arguments (e.g. habitat type) or is this framing one of the aspects where your study advances beyond those previous analyses, but not find any differences? If they only focused on climatic variables, I think you could move the description of this study to the earlier part of the introduction where you discuss climate (integrate lines 133-143 with/after the text in the paragraph starting in line 88).

Framing:

In the final paragraph of the introduction, you state that the main aim of this study is to determine "a difference in adult survival between habitat types". Accordingly, I think it would be helpful to already mention the relevant result in the first paragraph of the discussion. When you discuss these results (line 660ff), you could also link to the broader literature again to show how your set of findings (including your earlier studies) provide insights beyond these populations (see e.g. Galipaud, M., \& Kokko, H. (2020). Adaptation and plasticity in life-history theory: How to derive predictions. Evolution and Human Behavior, 41(6), 493-501.)

Minor typos:

There are some minor typos you might want to fix for the final version. I focused on the new and revised parts of the manuscript, so you might want go through the whole manuscript for another check:

Line 103: first letter not capitalised: if large scale climatic factors

Line 114: missing word: such [as] pair fidelity

Line 117: missing reference: Nord \& Nilsson 2016 Bio Let.

Table 4: final R2 value still has additional values after the decimal point

Line 380: I don't think you need the reference for U-CARE here in the results because you are already provide it in the methods

Line 431: missing space: quadratic effect

Line 518: change phrase: stronger dataset instead of strongest dataset

Line 641: change word: I think you mean provisioning rather than provisional

Preprint DOI: https://doi.org/10.1101/2021.01.28.428563

\section{Revision round \#1}

2021-03-01

\section{Author's Reply}

Download author's reply (PDF file)Download tracked changes file Dear Editor, 


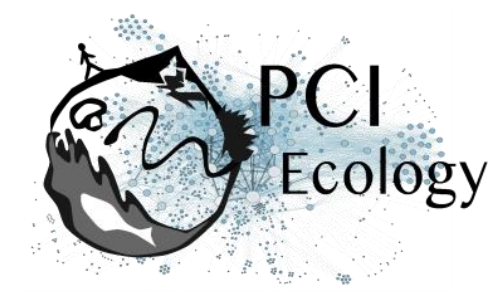

Attached you will find our response letter answering each reviewer comment, along with the new version of the ms which is also available on the BioRxiv server. If necessary we can also submit a tracked changes document, but since we have reformatted most figures and tables, it is very messy!

Best wishes,

Anne Charmantier

\section{Decision round \#1}

Dear Authors,

Your preprint entitled "Identifying drivers of spatio-temporal variation in survival in four blue tit populations"has now been reviewed and the reviewers' comments are appended below. As you will see, both reviewers consider your study to be sound and insightful, and I share their views. Yet they have several comments that need to be addressed carefully before I can recommend your preprint.

In particular, I agree with both reviewers that more detail should be provided in the manuscript to explain the potential links between the factors you investigate and survival. I think this will make the manuscript clearer and help readers to see how your findings might transfer to other populations or other species. From my perspective, as someone who does not know a lot about your study species of blue tit, I think it could also help to have a paragraph in the introduction that briefly explains some key parameters of the species as they relate to survival (e.g. seasonal breeder, non-migratory but swarm forming in the winter?, when do individuals of which sex disperse how far?, is the highest mortality in the winter, during incubation in the nest, during feeding from predation?, what is the maximum longevity?). Having this information might help to explain better in the introduction why you chose these various factors and how they could potentially shape survival in the different populations. In turn, I think it would help if the introduction is focused only on those factors that are central to your study: for example, the abstract mentions boldness which has no direct relation to your study setup.

I have an additional comment about the presentation of the approach and results in the tables and figures. There is a lot of information, but it is sometimes difficult to understand what information there is to extract for someone not as familiar with the setup as you.

Table 1: Is this supposed to be a supplement? It currently appears at the end of the manuscript, and you later focus on the more specific models rather than presenting the full breadth of results from all the models.

Table 3: There are some formatting issues in the pdf. I also wonder whether you want to set the number of digits after the decimal points (e.g. have four digits) to make comparisons easier.

Table 5: I think it would be interesting to have your results here as well for comparison. You could also add a column that explains whether each site is deciduous or evergreen.

Figure 1: Could you maybe include photographs that illustrate the forest conditions at the four sites?

Figure 2: The label for the fourth population E-Muro in red is missing. Maybe you could combine this figure with the additional illustrations of year-by-year variation shown in Figure 3 and Figure 4a.

Figure 3/4a: As mentioned above, the year-by-year trends on their own are not particularly informative. I think they gain their information from the comparison with the other trends, to compare the extent of variation in each and whether yearly fluctuations might align.

All the comments focus on adding information to the manuscript that presumably was already part of your decision processes. I am looking forward to read a new improved version of the manuscript to eventually recommend it.

Preprint DOI: https://doi.org/10.1101/2021.01.28.428563 


\section{Reviewed by Ana Sanz-Aguilar, 2021-02-19 12:25}

The manuscript "Identifying drivers of spatio-temporal variation in survival in four blue tit populations" analyzes four long term databases to understand the temporal variations in survival probabilities in relation with environmental conditions and density dependence. The scientific background is sound and the methods are robust and correctly implemented. I'm very surprised with the discrepancies with the previous analyses of Grosbois et al. The authors have made a good job re-analyzing the same time window to understand the discrepancies, but again results are different. As the only difference is the removal of experimental birds from the database, is it possible that these birds could be more affected by environmental conditions for any reason? (I recognize that I find this explanation weird but I have no alternative suggestions). In any case, I think that that the current analyses is better conducted that the one of Grosbois (I think that removing experimental birds is the correct decision) and the databases are longer and thus more suitable to explore factors affecting the temporal variations of survival. As a general suggestion I recommend the authors to check the beta estimates of covariates to be sure if the effect is positive or negative (if the $95 \% \mathrm{Cl}$ do not include zero) and to provide R square values (the percentage of temporal variance explained by covariates) for all the analyses.

Specific comments:

Figure 2. E-Muro is lacking in the Figure legend. I recommend to specify that model 18 include the effects of "age+pop.year" in survival.

Line 25. I suggest to indicate that populations "can" display lower survival. This is true for blue tits but may differ for other species/populations. As this is a general statement I suggest including the word "can" or "may".

Lines 26-27. Maybe the authors could indicate "adult blue tits" or "breeding blue tits". As age class is included among the effects tested it seems without further information that the analyses could deal also with juvenile survival, which is not the case.

Line 93. Please could you indicate in brackets the age classes considered?

Line 114. Please could you summarize the mechanisms provided/suggested by Grosbois et al to explain survival variation in Mediterranean populations of blue tits as a function of Sahel rainfall? How this index could influence climate in the Mediterranean is neither explained in methods (line 167)

Lines 199-204. Population density index. Sorry I cannot understand how the index was calculated. Only the next boxes present all the years were considered (e.g. 26 in E-Pririo) or different number of nest boxes were considered for different years?

Line 213. I suggest changing "censored right" by "right censored"

Line 226. It should be Fig $4 \mathrm{~b}$ instead Fig $3 \mathrm{~b}$.

Line 255. I think that model selection should be the same for all the covariates, not using the AIC for breeding density and the p-ANODEV for the others. I suggest to use all of them and discuss them at the light of R2 value that indicates the percentage of temporal variation explained by the covariate and finally (when there could be doubts) the authors could check the $95 \% \mathrm{Cl}$ of the beta estimate corresponding to the covariate slope to check if limits include zero.

Table 2. Here recapture probability P should be in capital letters to be consistent with the general notation in the manuscript.

Line 298. It should be Figure 3, instead Figure 3A.

Table 3. Why the model Phi (age+pop+q_winterMOI) is not included? 


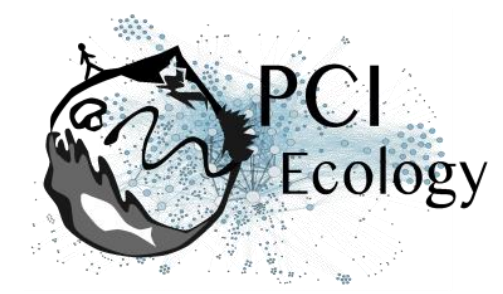

Line 351 . I recommend checking the $95 \% \mathrm{Cl}$ of the beta estimate to be sure that population density had a negative correlation with survival.

Line 449. Include the reference Blondel et al. 1992 after "see Table5".

Line 463. I think that here, as breeding density was not significant via ANODEV test it would be better to discuss the percentage of temporal variation explained by this covariate and also the $95 \% \mathrm{Cl}$ of the beta estimate (see previous comments).

\section{Reviewed by Vicente García-Navas, 2021-02-28 11:51}

This study relies on an impressive dataset (almost 40 years of study), which has allowed us to better know this species, the blue tit, and gain insight into key aspects within the field of Evolutionary Ecology, such as local adaptation or phenotypic plasticity. Some studies derived of this long-term research program are frequently included as textbook examples of adaptation to environmental heterogeneity, and exemplifies the peculiarities of the Mediterranean region. Prof. Anne Charmantier has taken the baton from the renowned Prof. Jacques Blondel and now she leads the team. The Montpellier group continues to produce high-quality science and contribute to the blue tit being considered the "lab rat" of field studies in Evolutionary Ecology. In this study, Bastianelli et al. use Capture-Mark-Recapture modelling to investigate the effects of climate (at both regional and local scale), breeding density, sex, and age on adult survival in four blue tit populations of Southern France. Overall, I find the study clear, concise and well executed, and the methodology is sound and solid. In my opinion,

the manuscript is generally clearly written and appropriately and thoroughly referenced; very minor language editing would be helpful throughout. My comments are generally minor and offered more to stimulate discussion than being critical. Despite the results are negative (no effect on climatic conditions on survival probability), I think this is a nice contribution, and the authors should move ahead with confidence. I only have some minor points which could help to further improve this paper:

Specific comments:

Page numbers missing.

Abstract:

L23 Habitat "naturalness" sounds odd. I'd use level of anthropogenization or something similar instead.

L24-25 I would say that 'populations whose individuals...' as talking about the behavior of a population as a whole is somehow controversial. Populations do not exhibit behavior syndromes, individuals do.

L38-39 Please, provide the (potential) reason for this decline. It is not clear if these two populations belong to the same habitat type (e.g., evergreen forest) or locality.

Introduction:

L46 Please, add Oxford (serial) comma after "trends".

L66 I would add a few examples of species or populations with strong density-dependent effects on their dynamics.

Material \& Methods:

L198 The number of nestboxes is rather low in all the studied populations in comparison with other studies in which the number of boxes typically oscillates between 80 and 100 . I am just curious about the mean occupation rate and the size of these woodlands (i.e., the number of breeding pairs per ha) (I am sure these details are given in previous studies carried out by the team but I think this info is appropriate for the purposes of the present study). In addition, besides occupation rates, it would be interesting to know if these (concrete?) nestboxes are used by other species -e.g., great tits, crested tits, nuthatches, rock sparrows-, with 


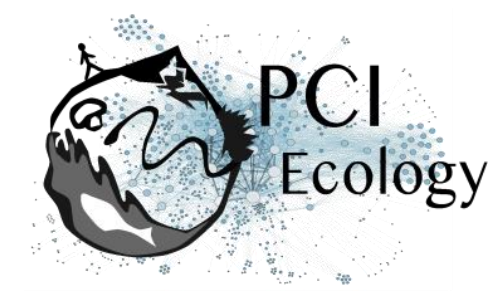

which blue tits compete for nesting places (cavities). Also, it would be interesting to know if nestboxes are protected against potential predators (snakes, weasels, woodpeckers) and if it -predation rates- constitutes a negligible/anecdotal factor or not at a population level.

L238 examined instead of "tested".

Results:

Fig.1: color coding for one locality (E-Muro) is missing. I find this figure a bit crude (too Excel-like) and a bit of elaboration would be more than timely.

L291-293 Intriguing. I wonder if this is related to differences in sex roles during the breeding period between habitats (e.g.; higher male provisioning effort in evergreen forests).

Fig. 3: I would superimpose a Generalized Additive Model (GAM) in each panel, which would allow to derive a trend across time (if any). I think this time series analysis would be more informative than the raw data.

L336 Suggestion: "[...] which differed between the Corsican (E-Pirio, D-Muro, E-Muro) and the mainland (DRouviere) populations".

L325. I would include a figure to illustrate the observed negative linear trend in survival in Rouviere and evergreen-Muro.

\section{L340 remaining populations?}

L342-343 This sentence is a bit misleading for the reader. Could you reformulate it a bit?

L343-343 Suggestion: There was found a linear temporal trend for population breeding density in the populations located at Muro.

\section{Discussion:}

L405-406 I agree with this explanation. However, it has been shown that parents contributions to brood care may vary across populations. Some studies have found that female blue tits feed their young less frequently than their mates regardless of the habitat type (evergreen or deciduous forest) (e.g., García-Navas \& Sanz, 2012. Condor, 114: 612-621).

L410-415 On this regard, I would be interesting to know if there are differences in terms of immigration rates (i.e., breeding adults not ringed as chicks in the focal population) among localities in order to know if different source-sink dynamics between the four study sites and the surrounding natural populations (birds breeding in tree cavities) may play a role in explaining the observed variation in recapture probabilities. I think authors may refer here to previous studies in which the studied sites are thoroughly described (e.g., Blondel et al. 2006, BioScience, 56: 661-673).

L424 Now, I think my previous comment on nest predation rates is especially opportune.

L463 Authors indicate that the effect of breeding density on adult survival was only detected in the two evergreen populations. However, they do not provide a rationale to explain this finding. It is a bit disappointing as (IMHO) the authors have enough information based on decades of work in this study system to discuss more in depth about why the effect of density is particularly noticeable in this habitat type (e.g., different local conditions: lower availability of food resources; larger foraging distances and territory sizes, etc.). Furthermore, I wonder if nestling mass determines survival probability in these populations. Do chicks fledge in worse conditions in sclerophyllous forests? If so, does nestling condition have a significant impact on survival? For instance, in a study with great tits, Rodríguez et al. (2016; Ecology \& Evolution, 6: 4458-4467) reported that large and early chicks have a greater first-year local survival probability. In the same population 


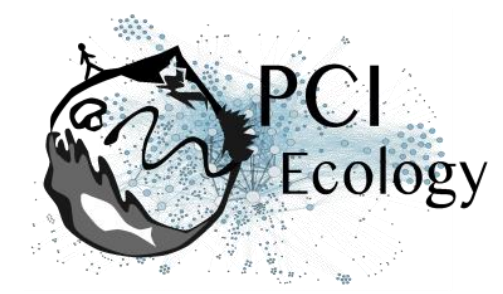

(located in a Mediterranean environment, orange groves in Valencia), Greño et al. (2008; Journal of Avian Biology, 39: 41-49) suggested that post-fledgling survival increased with mass, and decreased with temperatures during the nestling period.

L468-470 This is true, however I think authors could easily provide more details about: i) the density of nestboxes per ha, ii) the density of boxes occupied by blue tits per ha (I would expect a lower density of breeding pairs in the evergreen forests), iii) the density of boxes occupied by other competitor species per ha, iv) the average number of nests predated per year in each population, and $v$ ) the number of immigrants recruited in each population per year. This info would be very helpful in order to better understand the different local conditions to which blue tits must face in each of the four study sites. Thus, I suggest including a table with these five descriptive parameters for each population if possible.

L503-515 This result is striking as one would expect a decline linked to a particular habitat type (evergreen or deciduous) or locality. However, authors only found this pattern in one of the two localities at Muro, and the two localities displaying a decreasing linear trend belong to different habitat types (E \& D)! This certainly makes it difficult to draw any kind of conclusion.

Conclusions (last paragraph): Instead of highlighting the main drawbacks of the study, I would try to highlight its strengths. I think authors should try to improve the "home-take message". I found this last paragraph a bit vague; this study comprises a unique dataset and I think it deserves a better corollary.

Hopefully some comments will be useful to further improve the manuscript. Good luck with the peer-review process.

Best regards,

Vicente García-Navas 